\title{
Inhibition of wave-driven two-dimensional turbulence by viscoelastic films of proteins
}

\author{
N. Francois, H. Xia, H. Punzmann, T. Combriat, ${ }^{*}$ and M. Shats ${ }^{\dagger}$ \\ Research School of Physics and Engineering, Australian National University, Canberra, Australian Capital Territory 2611, Australia
}

(Received 30 December 2014; published 26 August 2015)

\begin{abstract}
To model waves, surface flows, and particle dispersion at the air-water interface one needs to know the essential mechanisms affecting the fluid motion at the surface. We show that a thin film (less than 10-nm thick) of adsorbed protein dramatically affects two-dimensional turbulence generated by Faraday waves at the fluid surface. Extremely low concentrations $(\approx 1 \mathrm{ppm})$ of soluble proteins form a strong viscoelastic layer which suppresses turbulent diffusion at the surface, changes wave patterns, and shows strong resilience to the wave-induced droplet generation. Surface shear properties of the film play a key role in this phenomenon by inhibiting the creation of vorticity at the surface. The addition of surfactants, on the other hand, destroys the nanolayer and restores the fluid mobility.
\end{abstract}

DOI: 10.1103/PhysRevE.92.023027

PACS number(s): 47.27.tb, 45.20.Jj, 47.52.+j

\section{INTRODUCTION}

Minute amounts of soluble polymers can dramatically modify the flow properties in liquids [1]. The addition of polymers to a turbulent flow can reduce substantially the drag in a pipe, a phenomenon known as the Toms effect [2]. However, polymers can also excite fluid motion over a broad range of spatial and temporal scales at low Reynolds numbers, and generate elastic turbulence [3]. These macroscopic effects hinge on the coupling between the polymer microscopic conformation and the flow. The interaction of polymers with fluid-solid or fluid-fluid interfaces has also been recognized as an important factor in the emergence of non-Newtonian effects [4-9]. The air-water interface is of obvious importance in many natural processes [10]. Moreover, great stretches of the oceanic waters are covered by very thin (less than $1 \mu \mathrm{m}$ thick) films made of organic matter such as proteins, lipids, and surfactants [11-13]. The ability to predict quantitatively the influence of this organic skin on fluid transport along the surface is key to better understanding mixing at the sea-air interface, ocean-atmosphere exchange, or spray production $[12,14]$.

Similar to synthetic polymers, these very thin organic films exert substantial elastic stresses on the fluid surface which are not included in the standard equations of hydrodynamics [1]. During the last decades, surface rheology has made progress in the characterization of such stresses which are often studied by deforming an interface using simple planar waves $[15,16]$. Compared with the effect of wave damping by insoluble incompressible oil films [10], known for centuries as "oil over troubled water," the effects of soluble viscoelastic biofilms on complex waves is still a recent subject with many debated questions. Even less is known about the influence of such films on the motion of the fluid parcels which compose the waves. While the influence of surface active substances on wave hydrodynamics has attracted a lot of attention recently, we emphasize that the wave dynamics is actually the motion of a surface shape which can be substantially different from that of the fluid particles [17].

*Current address: Magistère de Physique Fondamentale d'Orsay, Université Paris-Sud, 91405 Orsay, France.

${ }^{\dagger}$ Michael.Shats@anu.edu.au
In this study, we ask the question: what is the influence of biofilms on wave-driven surface flows and fluid particle dispersion? Recent progress in visualization of the fluid particle motion in complex three-dimensional (3D) waves [17] and the recent discovery that 2D turbulence (in the sense of KraichnanKolmogorov) can be forced by waves [18-21] open up exciting opportunities to address this question and explore important aspects of the surface hydrodynamics of natural water.

Here, we show that the turbulent fluid motion driven by 3D nonlinear waves, namely, Faraday waves, is dramatically affected by microscopic concentrations of proteins. We
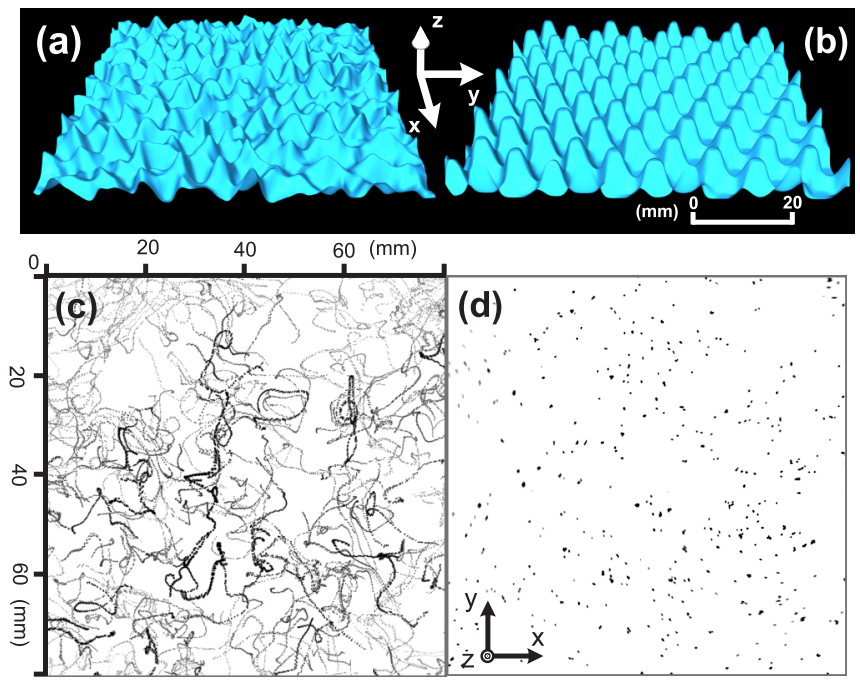

(e)

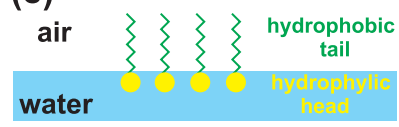

(f)

surface film BSA

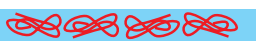

FIG. 1. (Color online) Perspective views of the measured Faraday wave field $\left(f_{0}=60 \mathrm{~Hz}, \epsilon=1.6\right)$ in (a) water with added surfactant and (b) in water with added 5 ppm of BSA. Top views (c) and (d) show floating particle trajectories corresponding to the wave fields (a) and (b) respectively. Particles (diameter $=50 \mu \mathrm{m}$ ) are filmed for $1 \mathrm{~s} \mathrm{(30} \mathrm{Faraday} \mathrm{wave} \mathrm{periods).} \mathrm{Schematics} \mathrm{of} \mathrm{an} \mathrm{interface}$ covered with (e) surfactant molecules or with (f) a film of adsorbed soluble BSA. 

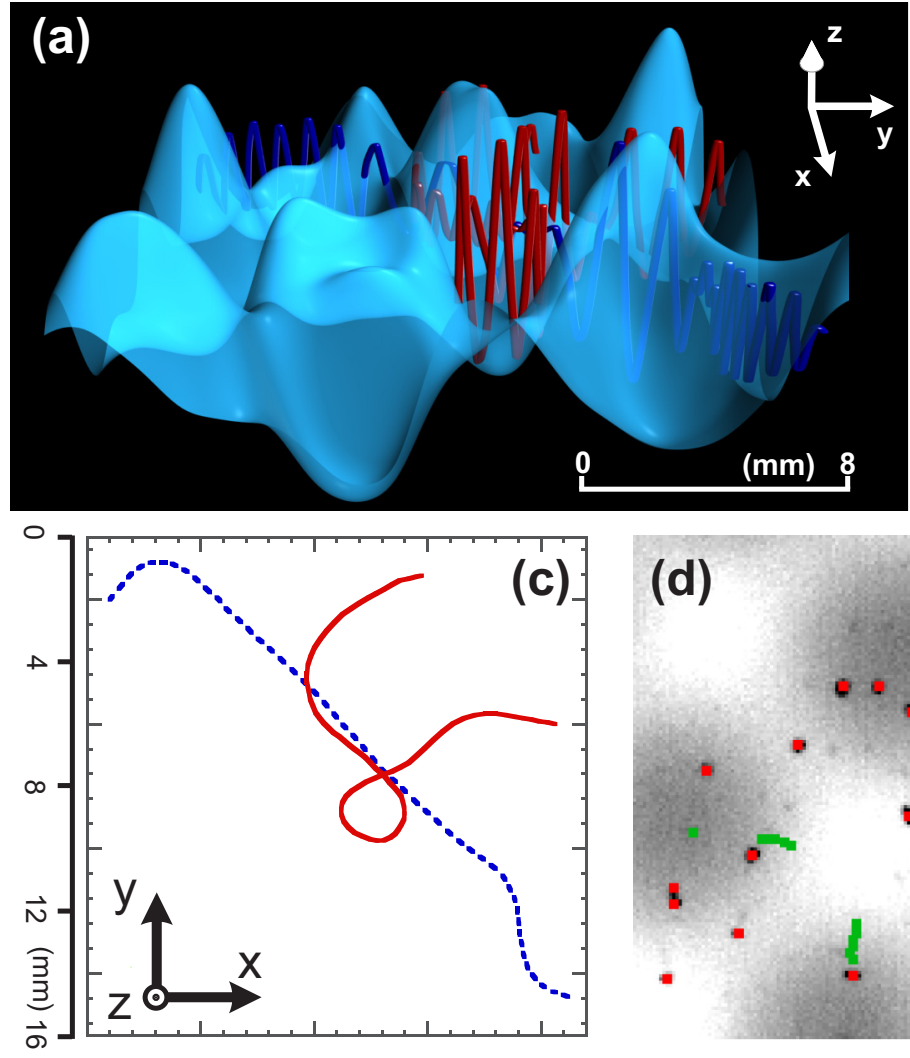

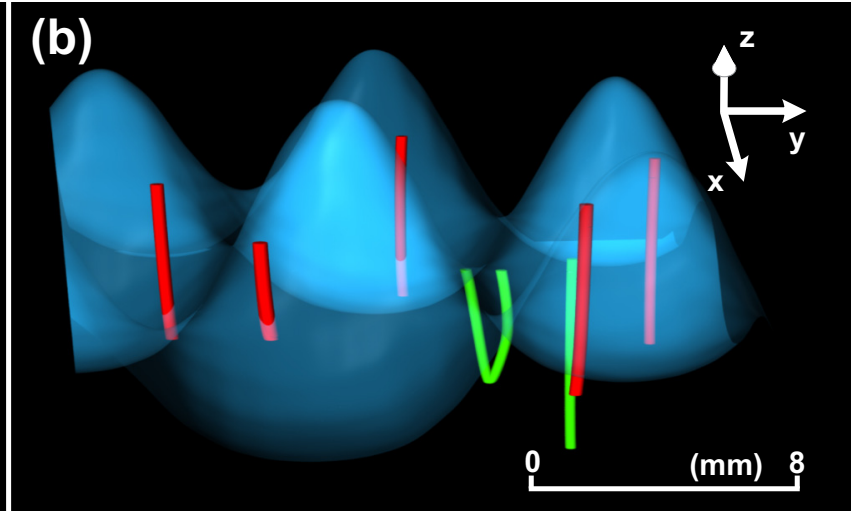

(e)

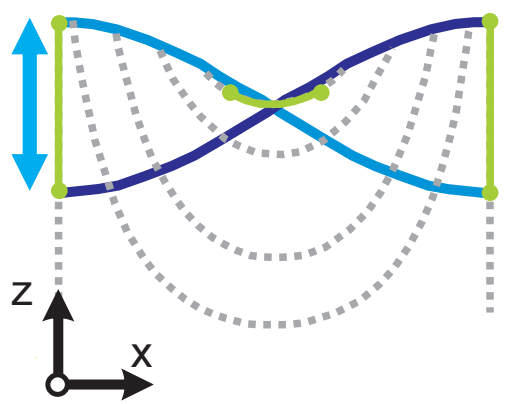

FIG. 2. (Color online) 3D particle trajectories of the fluid particles on the surface of (a) water with added surfactant and (b) water with $5 \mathrm{ppm}$ of BSA. Blue and red [dark gray, see top views in (c) and (d)] trajectories mark particles moving on the surface, while the green (light gray) trajectories correspond to particles $0.25 \mathrm{~mm}$ below the surface. (c) Top view of the trajectories shown in (a). (d) Top view of trajectories of particles at (red, dark gray) and below (green, light gray) the surface in the water+BSA case. Black and white blobs mark the wave crests and troughs respectively. (e) Schematic of theoretical particle trajectories [green (light gray) lines] and streamlines (grey dashed lines) in a 2D standing wave.

study the effect of soluble proteins, namely, bovine serum albumin (BSA), on 3D fluid particle trajectories and on the single-particle dispersion on the surface. We show that small concentrations of BSA ( $\geqslant 1 \mathrm{ppm}$ in weight) suppress the horizontal fluid motion by forming a viscoelastic surface film in which the fluid incompressibility assumption is violated. Although the film is less than $10 \mathrm{~nm}$ thick, it also greatly reduces the horizontal fluid motion under the surface, turning a disordered field of waves (1-6 mm high) into an ordered lattice. Among numerous rheological features of adsorbed layers of proteins, we show evidence that shear properties play a key role in the reported phenomenon by suppressing the creation of vorticity at the surface. If minute amounts of non-ionic surfactant is added to the fluid, it destroys the nanolayer and restores the fluid mobility.

\section{EXPERIMENTS AND RESULTS}

In these experiments, Faraday waves are formed at the surface of a liquid (distilled water with added surfactant or $\mathrm{BSA}$ ) in a circular container (diameter $=178 \mathrm{~mm}$ ) vibrated at a frequency $f_{0}=60 \mathrm{~Hz}$. Beyond a certain threshold for the vertical acceleration $a_{\mathrm{th}} \approx 0.6 \mathrm{~g}$, parametrically forced waves appear with a dominant frequency $f_{F}=f_{0} / 2=30 \mathrm{~Hz}$ and a wavelength $\lambda_{F} \approx 8.8 \mathrm{~mm}$. The depth of the liquid
(35 mm) is larger than $\lambda_{F}$. To compare experiments at different accelerations, we define the supercriticality parameter as $\epsilon=$ $\left(a-a_{\mathrm{th}}\right) / a_{\mathrm{th}}$. In water, Faraday waves appear as a disordered ensemble of oscillating solitons, or oscillons, that inject vorticity in the horizontal flow [18]. These horizontal vortices interact and spread energy over a broad range of scales in the process of the inverse energy cascade [22]. Ultimately the horizontal fluid particle motion on the surface resembles in detail that in 2D turbulence [19-21,23].

Recently, we reported that minute amounts of soluble proteins [Fig. 1(f)] turn a disordered wave field into a regular pattern of oscillons [24], Figs. 1(a) and 1(b). This effect is different from the wave suppression by immiscible oil films [10], since the BSA suppresses only the horizontal mobility of oscillons without affecting the wave heights. We also briefly mentioned that a droplet of surfactant [Fig. 1(e)] can annihilate this effect [26]. The mechanism behind these phenomena was not understood. Here we investigate what happens with the motion of the actual fluid parcels at and below the surface. First, the Lagrangian horizontal fluid motion at the surface is characterized by using standard 2D particle tracking techniques [21]. Figures 1(c) and 1(d) show horizontal trajectories of floating tracer particles. Figure 1(c) shows particle trajectories at the surface of water with added non-ionic surfactants [25]. In Fig. 1(d), the same visualizations 

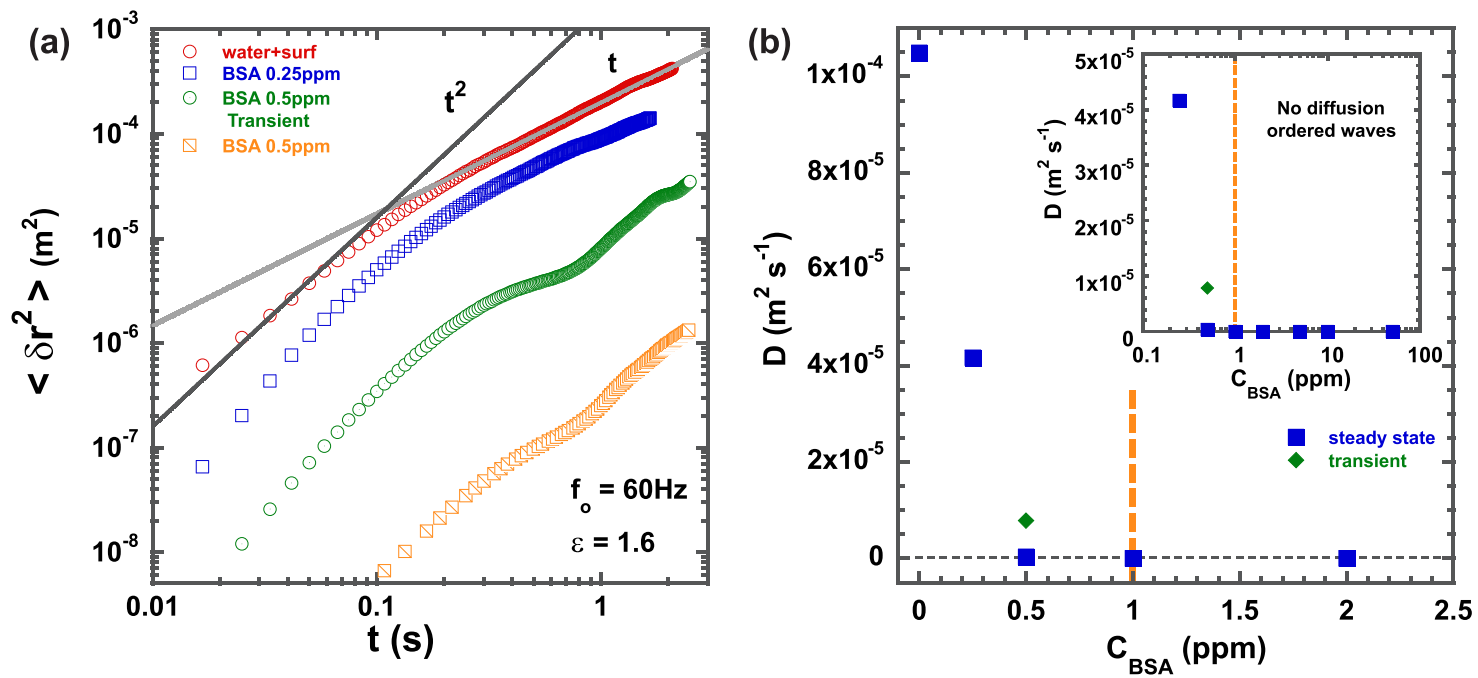

FIG. 3. (Color online) (a) $\left\langle\delta r^{2}\right\rangle$ as a function of time $t$ at different concentrations $c_{\text {BSA }}$ of BSA in water. (b) The steady-state diffusion coefficient $D$ on the water surface vs $c_{\mathrm{BSA}}$. Inset: same data in the lin-log scale. At low BSA concentration $\left(c_{\mathrm{BSA}}<1 \mathrm{ppm}\right)$, the diffusion coefficient was found to reach a steady state after a 10-min transient. An example of a transient measurement is included for $c_{\mathrm{BSA}}=0.5 \mathrm{ppm}$ (measurements performed after $2 \mathrm{~min}$ ).

are performed in water with added $5 \mathrm{ppm}$ (in mass) of BSA. Minute amounts of BSA stop the fluid particle motion. Therefore drastic changes of the surface "shape" coincide with the suppression of the horizontal motion of the fluid parcels that compose the waves.

To investigate the reason for such a strong suppression of the particle motion in the presence of BSA, we visualize 3D particle trajectories using a recently developed particle tracking method $[17,18]$. Figures 2(a) and 2(b) show several trajectories corresponding to the cases of Figs. 1(c) and 1(d). In water with added surfactant, the fluid motion on the surface is turbulent. The trajectories represent complex curves along which particles wander erratically in a succession of "traps" and "flights" over the whole wave field [18]. Trajectories are dramatically different if instead of surfactant, microscopic quantities of BSA are added. In this case all trajectories on the water surface [shown in red (dark gray) in Figs. 2(b) and 2(d)] are straight vertical lines. However, particles about $0.25 \mathrm{~mm}$ below the surface [green (light gray) trajectories in Figs. 2(b) and 2(d)] show strictly vertical motion near the wave crests, and pendulum-type oscillations near the wave nodes. These subsurface particles stay confined within the wave period.

The Faraday wave pattern in the presence of BSA is close to the ideal square lattice, or a perfect crystal made of standing oscillons, Fig. 1(b). In 2D standing waves, as has been demonstrated experimentally $[27,28]$ and theoretically [29], particles perform the pendulum-type motion near the wave nodes, while near the wave trough or crest particles oscillate vertically. Similar trajectories have been measured recently in 3D Faraday wave crystals formed in a highly viscous Newtonian liquid (glycerol + water solution) [18]. This pendulum-like motion is a signature of the incompressibility of the liquid.

In contrast, in the presence of BSA, only the particles below the surface show a motion similar to that in a viscous Newtonian liquid. All surface particles oscillate strictly vertically regardless of their position, Fig. 2(d). Such anomalous trajectories of fluid particles violate the incompressibility assumption and uncover the presence of a very thin gelatinous layer of adsorbed BSA possessing viscoelastic properties. It is known that BSA and other proteins adsorbed at the air-water interface form viscoelastic films [Fig. 1(f)] as thin as $10 \mathrm{~nm}[15,30,31]$. Despite the availability of rheological surface characteristics for BSA, very little is known about its effects on the surface hydrodynamics, such as 3D nonlinear waves and wave-induced fluid particle dispersion.

First, we consider the mean-squared displacement $\left\langle\delta r^{2}\right\rangle=$ $\left\langle|\vec{r}(t)-\vec{r}(0)|^{2}\right\rangle$ of a fluid particle at the wave surface, where $\vec{r}(t)$ is the horizontal position vector of the particle at time $t$. If Faraday waves are formed in water with added surfactant, a 2D turbulent flow is generated [20]. The theory of single fluid particle dispersion driven by turbulent flows dates back to Taylor [32] and it reads

$$
\begin{gathered}
\left\langle\delta r^{2}\right\rangle=\tilde{u}^{2} t^{2}, \quad t<T_{L}, \\
\left\langle\delta r^{2}\right\rangle=2 D t, \quad t>T_{L},
\end{gathered}
$$

where $\tilde{u}^{2}$ is the velocity variance, and $T_{L}$ is the integral time of the Lagrangian velocity autocorrelation function. Recent experiments showed that $D \approx \sqrt{\tilde{u}^{2}} L_{F}$ in $2 \mathrm{D}$ turbulence, where $L_{F}$ is the energy injection scale of turbulence [21]. In Faraday wave-driven turbulence $L_{F} \approx \lambda_{F} / 2$. The two regimes of the mean-squared displacement, also known as ballistic and diffusive regimes, can clearly be identified in Fig. 3(a). This figure also shows the effect of BSA on $\left\langle\delta r^{2}\right\rangle$ for various concentrations $c_{\mathrm{BSA}}$. The vertical acceleration $a$ in these experiments is fixed at a supercriticality of $\epsilon=1.6$. A noticeable reduction of the particle mobility is observed at $c_{\mathrm{BSA}}$ as low as $0.25 \mathrm{ppm}$. The diffusion coefficient $D$ at this concentration is reduced by almost a factor of 3 in comparison with the water + surfactant case, Fig. 3(b). Above 0.5 ppm, $D$ drops dramatically. It is initially (transient point) one-tenth the value in the water + surfactant, and it is eventually reduced by two orders of magnitude after a several minute transient. At 

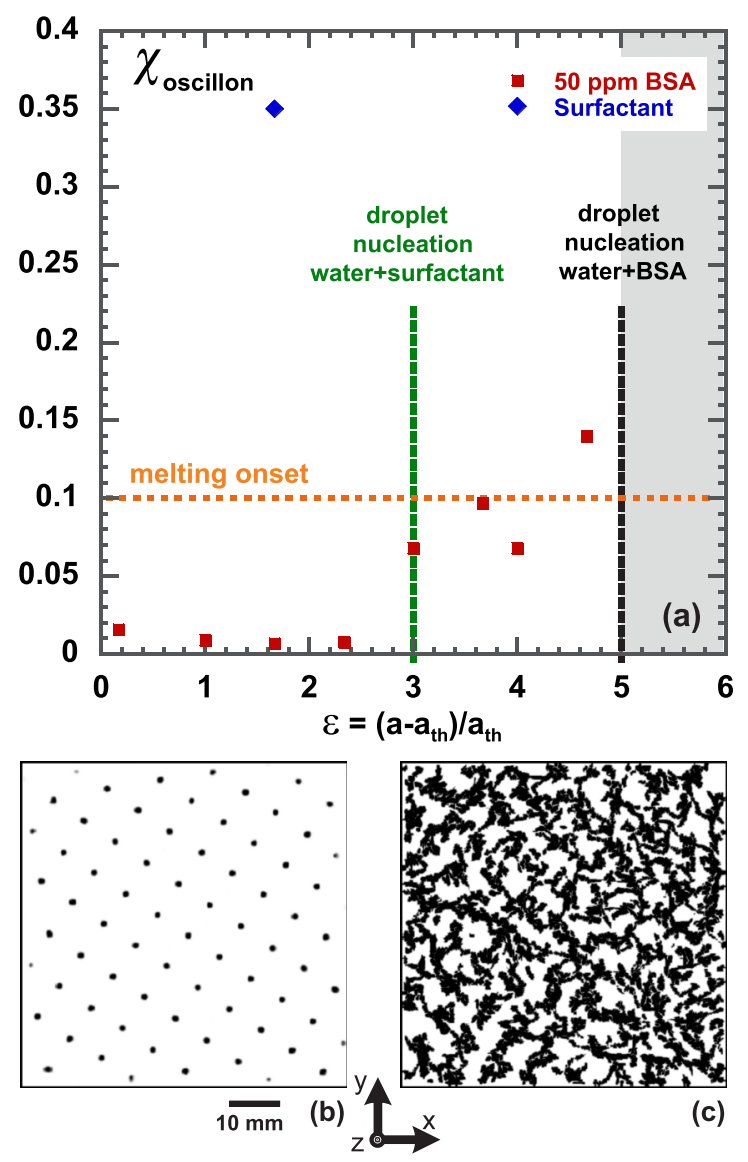

FIG. 4. (Color online) (a) Oscillon mobility $\chi_{\text {osc }}$ within the lattice vs the supercriticality parameter $\epsilon$ with added BSA or with added surfactant. $\chi_{\text {osc }}$ is averaged over 50 Faraday periods and 1000 oscillons. Oscillon trajectories were measured over 50 Faraday periods in (b) water+BSA and (c) water + surfactant at $\epsilon \approx 1.6$.

$c_{\mathrm{BSA}} \geqslant 1 \mathrm{ppm}$, no diffusive motion could be measured. The range of the BSA concentrations affecting horizontal diffusion is similar to the one identified in rheological measurements of the shear properties of BSA films [30]. The viscoelastic properties of BSA films depend on the protein concentration; the onset of elastic and viscous stresses was measured at a concentration of $\approx 0.2 \mathrm{ppm}$. Remarkably, the surface diffusion coefficient $D$ shows the same level of sensitivity to the presence of BSA.

Second, we evaluate quantitatively the effect of BSA on the ripples motion. To study oscillon mobility, we employ Particle Tracking Velocimetry techniques to track the motion of oscillon maxima in phase-synchronized movies. Each oscillon crest is identified with an algorithm that detects local maxima in the field of view. The trajectories of oscillon crests are reconstructed by using the particle tracking algorithm. We quantify the horizontal mobility of oscillons with the parameter $\chi_{\text {osc }}$ defined as

$$
\chi_{\mathrm{osc}}=\sqrt{\left\langle\left|\vec{r}_{i}(t)-\left\langle\vec{r}_{i}\right\rangle\right|^{2}\right\rangle_{i, t} / \lambda_{F}^{2}},
$$

where $\vec{r}_{i}(t)$ is the position vector of the oscillon $i$ at time $t$. This quantity is similar to the Lindemann ratio which has been used to characterize the melting transition in crystalline solids (e.g., [33]) and ordered patterns of oscillons in vibrated granular layers [34]. The melting onset of ordered patterns has been reported to occur for $\chi_{\text {melt }} \approx 0.1$ [34]. In the range $0<\epsilon<3$, $\chi_{\text {osc }}$ is low and almost constant [Fig. 4(a)]. In this case, the oscillon lattice is highly ordered and extremely stable in time [Fig. 4(b)]. Beyond $\epsilon=3$, the oscillon horizontal mobility increases abruptly and the lattice is more and more disordered. $\chi_{\text {osc }}$ becomes larger than $\chi_{\text {melt }}$ just before the onset of droplet nucleation. A drop of added surfactant to a water+BSA fluid enhances dramatically the oscillon mobility [Fig. 4(c)], destroys the regular patterns of waves at low $\epsilon$, and reduces substantially (to $60 \%$ ) the threshold for droplet nucleation. This is because surfactants disrupt interfacial protein-protein interaction by displacing proteins from the surface or by introducing new surface-active molecules [15,35].

\section{DISCUSSION AND CONCLUSIONS}

A layer of BSA is a gridlike network possessing nontrivial rheological characteristics [15]. The surface rheology determines several viscoelastic properties related to specific deformation of the surface of the liquid, such as a dilatational surface elasticity which characterizes the resilience to dilation deformation, and a shear modulus with respect to shearing deformation $[15,30,36]$. In our experiments these two deformation mechanisms are coupled, the fluid surface is dilated by the waves, and the wave-driven fluid motion generates strong shear in the horizontal plane [see Figs. 1(c) and 5(b)].

BSA films show a dilatational resistance comparable to that of surfactants used in these experiments $[16,36]$. At concentration $c_{\mathrm{BSA}} \geqslant 1 \mathrm{ppm}$, the shear viscosity of BSA films is orders of magnitude larger than that of surfactants [16,30]. Wavedriven turbulence is observed in the presence of surfactants. These facts along with the behavior of the diffusion coefficient $D$ (Fig. 3) suggest that shear properties of the protein layer are the key parameters to understanding the suppression of the horizontal fluid motion in BSA water solutions interacting with Faraday flows. To further test the prime role played by the shear viscosity and to decouple effects related to dilatational deformation from those induced by shearing, we performed experiments in electromagnetically driven flows (EMFs) [37]. In that setup, the fluid surface is not dilated by surface waves and the surface motion is generated by the Lorentz force, creating an array of horizontal vortices [Fig. 5(c)]. These vortices create strong in-plane shearing (precisely strong shearing and strong elongational stretching) [Fig. 5(a)]. However, by adding about 2 ppm of BSA we completely suppress the fluid motion on the water surface [Fig. 5(d)].

On a related matter, it has recently been observed that the addition of long flexible polymers in two-dimensional turbulence produced in fast-flowing soap films significantly reduces the level of the velocity fluctuations [38]. In the present study, the effect of a nanolayer of BSA on Faraday wave-driven turbulence is even more dramatic since it totally extinguishes the horizontal fluid motion. The presence of BSA inhibits the creation of a lattice of vortices at the wave surface which is the fueling mechanism of 2D turbulence [18]. The regions between vortices, also called saddle points, are characterized by strong deformation rate of the fluid elements [Fig. 5(a)]. The large shear viscosity of the BSA film [30] might strongly hinder the 


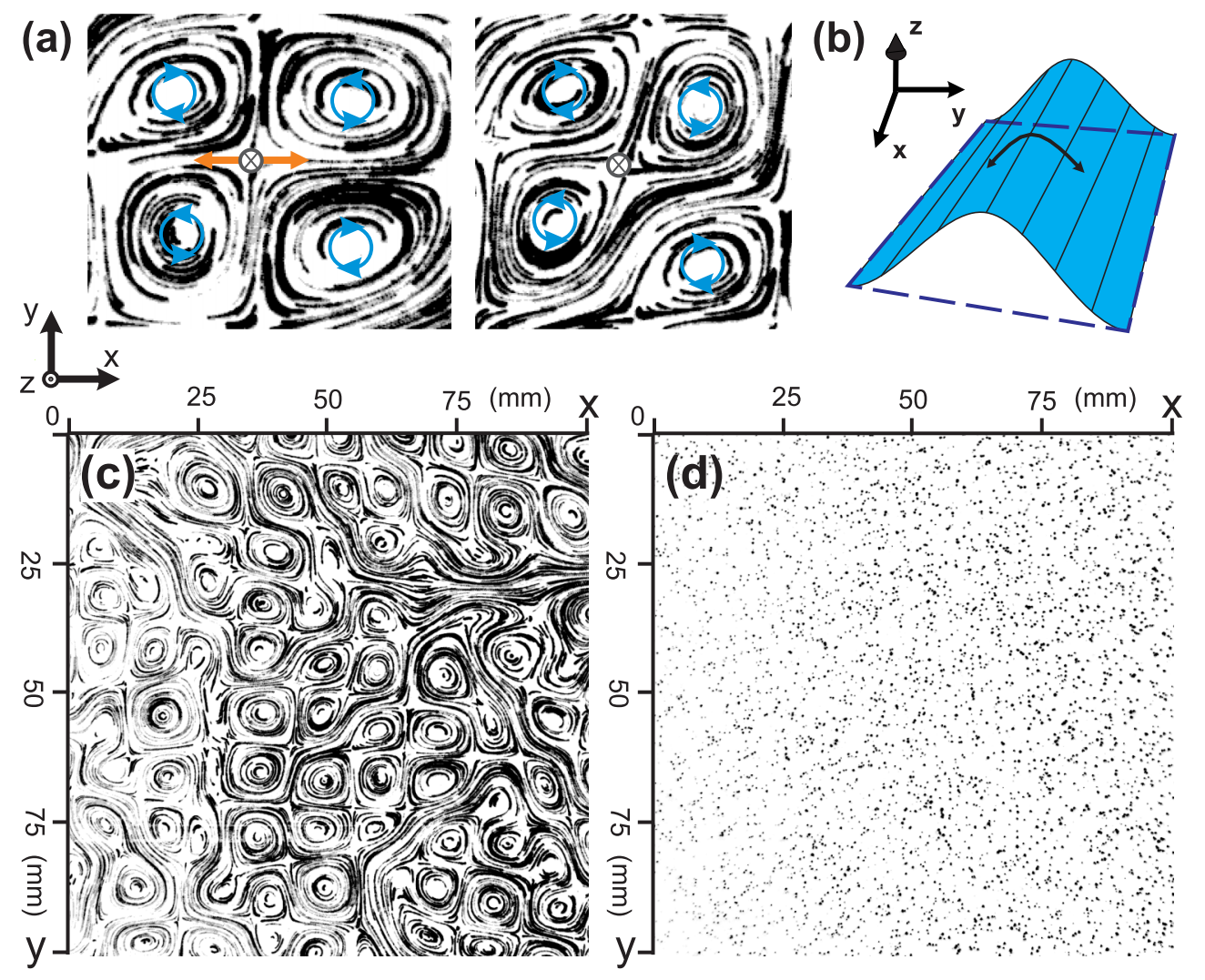

FIG. 5. (Color online) (a) Two close-ups of (c) showing the fluid deformation (both shearing and stretching) induced by four counter-rotating vortices in the electromagnetically driven flow (EMF). The white dot with a cross locates the saddle point in the velocity field. (b) Schematics of the surface dilatation induced by a wave. (c) Trajectories of floating particles in EMF at low electrical forcing in the presence of surfactants. Particles are tracked for $3 \mathrm{~s}$. In this case, a periodic lattice of vortices is produced. (d) Same configuration as in (c) but in the presence of 2 ppm of BSA.

formation of these saddle points and therefore suppress the creation of vorticity. Quantitatively, this can be understood by comparing the characteristic shear rate at the forcing vortex scale $\gamma_{F}$ with the characteristic relaxation time $\tau$ of the BSA film at very low concentration $\left(c_{\mathrm{BSA}} \approx 0.25 \mathrm{ppm}\right)$ [30]. We estimate that $\gamma_{F} \tau \approx 10$; a value larger than 1 indicates the presence of strong elastic effects.

The above rationale is consistent with our observations that the BSA film seems not to affect the vertical oscillatory component of the fluid motion in Faraday waves and that such a film also suppresses the formation of a lattice of vortices in electromagnetically driven flows. However, the addition of surfactants restores the vorticity creation. The reported results might open up new perspectives for the understanding and the control of horizontal mixing and spray production at the sea-air interface $[13,14,39]$.

\section{ACKNOWLEDGEMENTS}

The authors thank M. Roché and V. Craig for useful discussions. This work was supported by the Australian Research Council's Discovery Projects funding scheme (DP150103468). H.X. acknowledges support by the Australian Research Council's Future Fellowship (FT140100067).
[1] R. G. Larson, The Structure and Rheology of Complex Fluids (Oxford University Press, Oxford, 1998).

[2] B. A. Toms, Proceedings of the International Congress on Rheology (North-Holland, Amsterdam, 1949).

[3] A. Groisman and V. Steinberg, Nature (London) 405, 53 (2000).

[4] A. Groisman and V. Steinberg, Nature (London) 410, 905 (2001).

[5] I. Procaccia, V. S. Lvov, and R. Benzi, Rev. Mod. Phys. 80, 225 (2008).

[6] N. François, D. Lasne, Y. Amarouchene, B. Lounis, and H. Kellay, Phys. Rev. Lett. 100, 018302 (2008); N. François, Y.
Amarouchene, B. Lounis, and H. Kellay, Europhys. Lett. 86, 34002 (2009).

[7] J. Eggers and E. Villermaux, Rep. Prog. Phys. 71, 036601 (2008).

[8] Y. Amarouchene, D. Bonn, J. Meunier, and H. Kellay, Phys. Rev. Lett. 86, 3558 (2001).

[9] F. Ingremeau and H. Kellay, Phys. Rev. X 3, 041002 (2013).

[10] H. Hünherfuss, in Marine Surface Films, edited by M. Gade, G. M. Korenowski, and H. Hünherfuss (Springer-Verlag, Berlin, 2006). 
[11] The Sea Surface and Global Change, edited by P. S. Liss and R. A. Duce (Cambridge University Press, Cambridge, England 1997).

[12] O. Wurl and M. Holmes, Marine Chem. 110, 89 (2008).

[13] J. M. Sieburth, in Air-Sea Exchange of Gases and Particles, edited by P. S. Liss and W. G. N. Slinn (Reidel, Hingham, MA, 1983), p. 121.

[14] M. Cunliffe, A. Engel, S. Frka, B. Gašparovic, C. Guitart, J. C. Murrell, M. Salter, C. Stolle, R. Upstill-Goddard, and O. Wurl, Prog. Oceanogr. 109, 104 (2013).

[15] M. A. Bos and T. van Vliet, Adv. Colloid. Interface Sci. 91, 437 (2001).

[16] D. Georgieva, A. Cagna, and D. Langevin, Soft Matter 5, 2063 (2009).

[17] H. Punzmann, N. Francois, H. Xia, G. Falkovich, and M. Shats, Nat. Phys. 10, 658 (2014).

[18] N. Francois, H. Xia, H. Punzmann, S. Ramsden, and M. Shats, Phys. Rev. X 4, 021021 (2014).

[19] A. von Kameke, F. Huhn, G. Fernández-Garcia, A. P. Muñuzuri, and V. Pérez-Muñuzuri, Phys. Rev. Lett. 107, 074502 (2011).

[20] N. Francois, H. Xia, H. Punzmann, and M. Shats, Phys. Rev. Lett. 110, 194501 (2013)

[21] H. Xia, N. Francois, H. Punzmann, and M. Shats, Nat. Commun. 4, 2013 (2013).

[22] R. Kraichnan, Phys. Fluids 10, 1417 (1967).

[23] H. Xia, N. Francois, H. Punzmann, and M. Shats, Phys. Rev. Lett. 112, 104501 (2014).
[24] M. Shats, H. Xia, and H. Punzmann, Phys. Rev. Lett. 108, 034502 (2012).

[25] We use two different surfactants: a wetting agent from Ilford and N-dodecyl B-D-maltoside (C12G2) from Sigma-Aldrich.

[26] H. Xia, T. Maimbourg, H. Punzmann, and M. Shats, Phys. Rev. Lett. 109, 114502 (2012).

[27] A. Wallet and F. Ruellan, La Houille Blanche 5, 483 (1950).

[28] M. Umeyama, Phil. Trans. R. Soc. A 370, 1687 (2012).

[29] M. Ehrnström and E. Wahlén, J. Nonlinear Math. Phys. 15, 74 (2008).

[30] D. E. Graham and M. C. Phillips, J. Colloid. Interface Sci. 76, 240 (1980).

[31] L. G. C. Pereira, O. Theódoly, H. W. Blanch, and C. J. Radke, Langmuir 19, 2349 (2003).

[32] G. I. Taylor, Proc. London Math. Soc. s2-20, 196 (1922).

[33] V. M. Bedanov, G. V. Gadiyak, and Yu. E. Lozovik, Phys. Lett. A 109, 289 (1985).

[34] D. I. Goldman, M. D. Shattuck, Sung Joon Moon, J. B. Swift, and H. L. Swinney, Phys. Rev. Lett. 90, 104302 (2003).

[35] M. Roche, Z. Li, I. M. Griffiths, S. Le Roux, I. Cantat, A. Saint-Jalmes, and H. A. Stone, Phys. Rev. Lett. 112, 208302 (2014).

[36] D. E. Graham and M. C. Phillips, J. Colloid. Interface Sci. 76, 227 (1980).

[37] H. Xia, M. Shats, and G. Falkovich, Phys. Fluids 21, 125101 (2009).

[38] Y. Amarouchene and H. Kellay, Phys. Rev. Lett. 89, 104502 (2002).

[39] H. Lhuissier and E. Villermaux, J. Fluid Mech. 696, 5 (2012). 